\author{
Rita S. Dias ${ }^{1,2}$ \\ Roine Svingen ${ }^{3}$ \\ Bodil Gustavsson ${ }^{3}$ \\ Björn Lindman ${ }^{1,2}$ \\ Maria G. Miguel ${ }^{1}$ \\ Björn Åkerman ${ }^{3}$ \\ ${ }^{1}$ Chemistry Department, \\ University of Coimbra, \\ Coimbra, Portugal \\ ${ }^{2}$ Physical Chemistry 1 , \\ Center for Chemistry and \\ Chemical Engineering, \\ Lund, Sweden \\ ${ }^{3}$ Department of Chemistry and \\ Bioscience, Chalmers \\ University of Technology, \\ Göteborg, Sweden
}

\section{Electrophoretic properties of complexes between DNA and the cationic surfactant cetyltrimethylammonium bromide}

\begin{abstract}
We use agarose gel electrophoresis to characterize how the monovalent catioinic surfactant cetyltrimethylammonium bromide (CTAB) compacts double-stranded DNA, which is detected as a reduction in electrophoretic DNA velocity. The velocity reaches a plateau at a ratio $R=1.8$ of CTAB to DNA-phosphate charges, i.e., above the neutralization point, and the complexes retain a net negative charge at least up to $R=200$. Condensation experiments on a mixture of two DNA sizes show that the complexes formed contain only one condensed DNA molecule each. These CTAB-DNA globules were further characterized by time-resolved measurements of their velocity inside the gel, which showed that CTAB does not dissociate during the migration but possibly upon entry into the gel. Using the Ogston-model for electrophoresis of spherical particles, the measured in-gel velocity of the globule is quantitatively consistent with CTAB having two opposite effects, reduction of both the electrophoretic charge and DNA coil size. In the case of CTAB the two effects nearly cancel, which can explain why opposite velocity shifts (globule faster than uncomplexed DNA) have been observed with some catioinic condensation agents. Dissociation of the complexes by addition of anionic surfactants was also studied. The DNA release from the globule was complete at a mixing ratio between anionic and cationic surfactants equal to 1 , in agreement with equilibrium studies. Circular DNA retained its supercoiling, and this demonstrates a lack of DNA nicking in the compaction-release cycle which is important in DNA transfection and purification applications.
\end{abstract}

Keywords: Cetyltrimethylammonium bromide / DNA

DOI 10.1002/elps.200400182

\section{Introduction}

Complexation with cationic lipids is one strategy for delivery of DNA to cells. Binding of the lipids leads to compaction of the DNA coils and to a reduction of its surface charge. Both effects are believed to contribute to the facilitated uptake of the nucleic acids through the cellular membrane [1-5]. Methods to monitor size and charge of the complexes are therefore helpful tools in the process of screening protocols for packaging the DNA before in vivo experiments are performed. Commonly a titration series is made where the ratio $R$ between concentration of charges from cationic lipid and DNA-phosphate groups is varied, usually in a range that includes the point $R=1$ where the number of added positive and negative charges is equal. It is widely accepted that surfactants display a very strong

Correspondence: Professor Björn Åkerman, Department of Chemistry and Bioscience, Chalmers University of Technology, S-142 96 Göteborg, Sweden

E-mail: baa@chembio.chalmers.se

Fax: +46-31-7723858

Abbreviations: EB, ethidium bromide; sos, sodium octyl sulfonate associative binding with DNA inducing its compaction [68], aggregation [9], and precipitation [8, 10, 11]. The associative phase behavior presented by these systems and the absence of redissolution with the addition of an excess of surfactant lead to the assumption that the formed complexes in solution are neutral $[8,12]$.

Electrophoresis has been used to study the properties of lipoplexes because the mobility reflects their net charge. Mobilities in free solution have been used [13, 14] to quantify the decrease in net charge that results from the added positive lipid charges. Complete neutralization and even charge reversal (net positive charge) have indeed been attained by adding an excess of cationic lipids, with the crossover characteristically occurring at $R$-values between 1 and 3. Parallel analysis by electrophoresis in gels [13] showed that the complexes were retained in the well, but this was ascribed to that they were too large to enter the gel rather than to that they were neutral. The DNA-lipid complexes studied by Bhattacharaya and Mandal [15] did enter the gel, and they report a decreased velocity (mobility shift) compared to unmodified DNA due to a decrease in net charge upon lipid binding [15], but again charge-neutral complexes were not observed. The proposed explanation 
was that the (catioinic) fluorescent dye ethidium bromide (EB) used to detect the DNA zones may have low affinity for complexes with such high amount of cationic charges from the lipid. This is clearly possible since binding of cationic lipids is known to expel EB from DNA $[13,16]$.

In addition to reflecting changes in charge, the mobility shifts in gel electrophoresis can reveal changes in DNA coil size caused by surfactant binding. Dauty and Behr [17] reported that complexes with a dicationic detergent migrate faster than the uncomplexed DNA in spite of the positive charges added by the detergent. They ascribed this opposite mobility shift to a counteracting effect of the reduced DNA size upon complexation, from the native coil state to a globule-like complex. Gel electrophoresis is a potential method to study such conformational changes because medically interesting plasmids are in the size range where DNA coils can be size-separated through sieving in the gel pores [18]. In the Ogston model for sieving, the electrophoretic mobility $\mu=v / E$ is given by [19]

$$
\frac{\mu}{\mu_{0}}=\mathrm{e}^{-\frac{\pi}{4}\left(\frac{\mathrm{R}_{\mathrm{s}}}{\mathrm{R}_{\mathrm{p}}}\right)^{2}}
$$

where $\mu_{0}$ is the mobility in free solution (no gel), $R_{\mathrm{p}}$ the average pore size, and $R_{\mathrm{s}}$ is the effective coil size of the DNA molecules, which for native DNA is best described by the radius of gyration [20]. According to the Ogston model a decrease in coil size is thus expected to increase the DNA velocity exponentially, because the smaller coils will collide less frequently with gel fibers. The effect of a reduced electrophoretic DNA charge due to bound surfactant, on the other hand, will in this model be accounted for by a decrease in $\mu_{0}$.

The monovalent cationic surfactant, cetyltrimethylammonium bromide (CTAB), is one of the most wellcharacterized amphiphilic condensing agents for DNA. The thermodynamic properties of the complexes with dsDNA have been investigated in great detail. A binding isotherm for CTAB to DNA has been measured [6], and has been modeled [21] by the McGhee-van Hippel theory for cooperative binding to DNA. The phase diagram in absence and presence of added monovalent salt has been established [8], and the dissociation of the complex by anionic surfactant has been characterized [22]. Importantly, single-molecule fluorescence microscopy has been used to characterize the mechanism of both complex formation [6, 7] and dissolution [22] in terms of a coexistence between the coil and globule state that occurs in an intermediate range of $R$-values. However, the electrophoretic properties of complexes between DNA and CTAB have not been studied to our knowledge. Our aims were to (i) measure CTAB-induced mobility shifts of DNA in gels and compare with the Ogston model, (ii) ascertain if charge-neutral complexes do exist at high $R$-values, accounting for potential artefacts due to poor dye staining, and (iii) characterize the charge and conformation state of the DNA after it has been released from the CTAB complexes by addition of anionic surfactants. The last aspect is important because after delivery, the DNA must become accessible to the enzymatic machinery of the cell. Since lipid complexation is known to inhibit at least certain DNA processing enzymes such as DNAse, $[16,23]$ it is likely that the transfected DNA can become active only by efficient release from the lipid complex. In vitro such release can be accomplished by addition of anionic lipids, which binds the cationic lipid and release the DNA [8, 15, 24], and there are indications that such a mechanism may play a role also in vivo [25].

\section{Materials and methods}

\subsection{Materials}

CTAB was obtained from Sigma (St. Louis, MO, USA), sodium octylsulfate (SOS) from Merck (Darmstadt, Germany), and SDS from BDH Chemical Lda (Lisbon, Portugal), and were used as received. ds $\lambda$ DNA (48 504 bp) and ФX174 DNA (5386 bp) were from MBI Fermentas (Vilnius, Lithuania), T7 DNA (39 $937 \mathrm{bp}$ ) from Sigma. $\lambda$ DNA was used as received or cut with restriction enzyme Xhol to obtain two linear fragments of 33 and $15 \mathrm{kbp}$. These fragments, as well as $\lambda$ DNA itself, were heated to $65^{\circ} \mathrm{C}$ for $10 \mathrm{~min}$ before the electrophoresis experiments to break up hybridization between self-complementary ends. ФX174 DNA was used both in the circular and linear forms. The original sample contained about $80 \%$ in the supercoiled form and $10 \%$ each of nicked circles and linear form. Pure linear form was obtained by restriction cleavage at the unique site of Eco 1471 (MBI Fermentas). The DNA fluorescent probes, YOYO-1 and EB, were obtained from Molecular Probes (Eugene, OR, USA), agarose from Pronadisa (Madrid, Spain).

\subsection{Sample preparation}

Surfactant solutions were prepared by simple dissolution to the desired concentrations. All stock solutions and samples were prepared with the electrophoresis $50 \mathrm{~mm}$ TBE buffer ( $50 \mathrm{~mm}$ boric acid and $50 \mathrm{~mm}$ Tris, with $1.25 \mathrm{~mm}$ EDTA). The concentration of DNA was kept constant in each of the electrophoresis experiments and was equal to $20 \mu \mathrm{g} / \mathrm{mL}$ (61 $\mu \mathrm{m}$ phosphates) if not otherwise stated. The concentrations of cationic and anionic surfactants were varied in a titration series in each experiment. The mixing ratio $R=$ [surfactant]/[DNA] equals the ratio of surfactant and DNA charges, whereas $S_{\mathrm{R}}=\left[\mathrm{S}^{-}\right] /\left[\mathrm{S}^{+}\right]$is the molar 
ratio between the negative and positively charged surfactants concentrations. Except for a few experiments with poststaining of gel with EB after electrophoresis (see text), the DNA molecules were prestained with one YOYO-1 molecule per $40 \mathrm{bp}$. The DNA-dye samples were left to equilibrate at $50^{\circ} \mathrm{C}$ for $2 \mathrm{~h}$ before the CTAB was added, in order to obtain a homogenous distribution of the dye molecules among the DNA molecules [26]. After the surfactant was added, the samples were left to equilibrate for $30 \mathrm{~min}$ if not otherwise stated. To increase sample density for loading purposes $5 \%$ ficoll was added, whereas the commonly used loading dye bromophenol blue was avoided since we noted some interaction between the dye and CTAB. Final sample volumes were $20 \mu \mathrm{L}$ each. All experiments were conducted at $25^{\circ} \mathrm{C}$.

\subsection{Electrophoresis}

Electrophoresis was used to study both the condensation of DNA by cationic surfactants and the dissociation of the formed complexes by the addition of an anionic surfactant. The samples were run in submarine mode in $50 \mathrm{~mm}$ TBE buffer on a $1 \%$ agarose gel at $5 \mathrm{~V} / \mathrm{cm}$ constant field. For the samples prestained with YOYO-1, the band pattern after electrophoresis was obtained by simply scanning the gel using the fluorescence of the dye with $488 \mathrm{~nm}$ excitation and $530 \pm 10 \mathrm{~nm}$ bandpass emission filter. In the absence of YOYO-1, the gel was stained after electrophoresis with EB $(0.5 \mu \mathrm{g} \mathrm{EB} / \mathrm{mL} 50 \mathrm{~mm}$ TBE buffer) for $1 \mathrm{~h}$ before the scanning using $514 \mathrm{~nm}$ excitation and $610 \mathrm{~nm}$ long pass emission filter. The position of each zone was taken to be the location of the maximum intensity, because this gives a well-defined position also for the rather broad zones obtained at high mixing ratios.

\section{Results and discussion}

\subsection{Mobility shift due to CTAB-induced condensation of DNA}

Figure 1a shows the gel electrophoresis analysis of $5.4 \mathrm{kbp}$ linear $\Phi \times 174$ DNA complexed with increasing amount of $\mathrm{CTAB}$, at mixing ratios $R=[\mathrm{CTAB}] /[\mathrm{DNA}]$ between 0.1 and 150. It is seen that the DNA zone exhibits a reduction in velocity (a mobility shift) with increasing $R$, which indicates that the dominating effect of the binding of cationic CTAB is a reduction of the effective charge in agreement with earlier studies of binding of catioinic lipids to DNA [15]. Figure $1 \mathrm{~b}$ (squares) shows the velocity of the zone relative to that for the unmodified DNA, $v / v(R=0)$. The velocity decreases until $R=1.8$, after which it reaches a plateau with a finite velocity. Such a biphasic behavior is consistent with a decrease in electrophoretic charge as the amount of
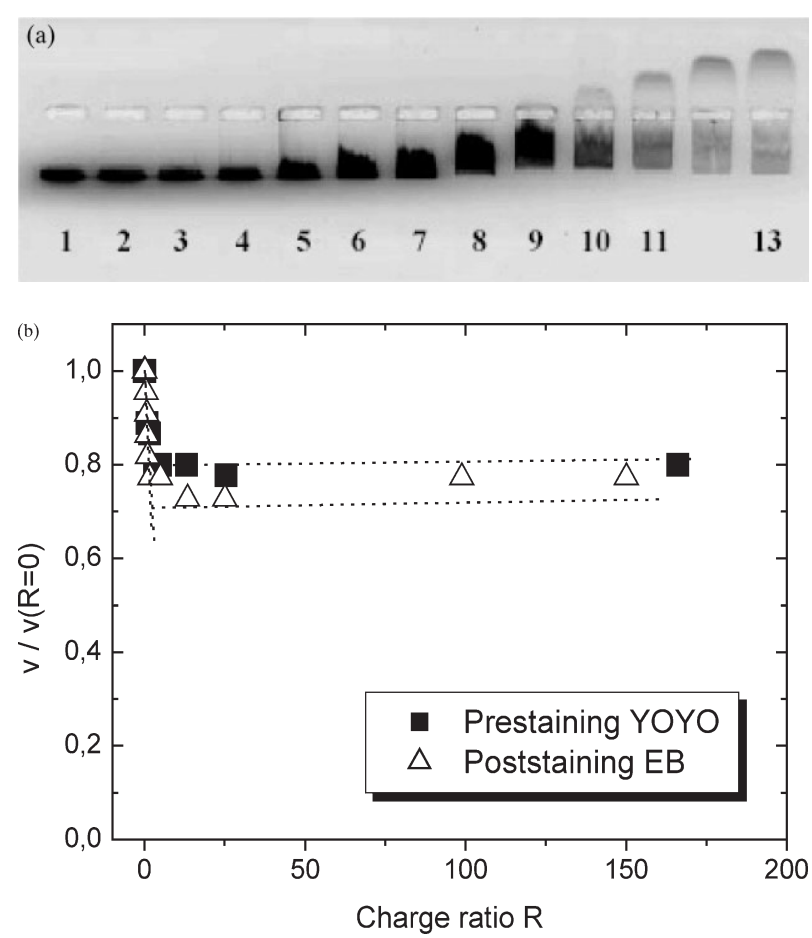

Figure 1. Mobility shift of DNA due to complexation with CTAB. (a) Electrophoresis of linear $\Phi \times 174$ DNA and its complexes with CTAB. Concentration of DNA is constant $(20 \mu \mathrm{g} / \mathrm{mL})$ and the mixing ratio CTAB to DNA phosphates, $R$ is equal to (from left to right): 0 (no surfactant), $0.1,0.2,0.5,0.7,0.8,1.0,1.7,5.0,13.4,25.1,98.8$, and 150.0. Prestaining of DNA with YOYO-1 at 1 dye/40 bp. (b) Relative velocity of CTAB-DNA complexes (compared to noncomplexed DNA) versus mixing ratio $R$ for YOYOprestained DNA (squares; from (a)) or with ethidium bromide post-staining (triangles) of the gel.

bound surfactant increases linearly with $R$ and then saturates approximately at $R=1.2$ [6]. Notably the DNA is migrating even for mixing ratios as high as 160 , so neutral complexes were not observed (at high $R$-values there are zones which do migrate towards negative electrode, but control experiments show that they do not contain DNA (see below)). It is also noteworthy that we observe one single zone that is gradually shifted as more and more CTAB is added, and not two discrete zones even at $R$-values where microscopy has demonstrated coexistence between coiled and globular DNA [6, 7]. This observation suggests that there is an interconversion between globular and coiled states on the time scale of hours characteristic for the electrophoretic analysis.

In the experiment illustrated in Fig. 1a, the DNA was prestained with the cationic dimeric cyanine dye YOYO-1. It has been shown [27] that the effects of YOYO binding on the electrophoretic properties of DNA itself is very small at our staining ratios of one dye per $40 \mathrm{bp}$, and secondly it has been 
reported [28] that this tetravalent catioinic dye remains bound to DNA after complexation with CTAB up to at least a charge ratio of $R=10$. In order to check to what extent the prestaining affects the process of CTAB-induced compaction, the experiment in Fig. 1a was repeated by complexing YOYO-free DNA with CTAB, and poststaining the gel with EB after the electrophoretic analysis. Figure $1 \mathrm{~b}$ (triangles) shows that the mobility-shift behavior is similar to those observations with prestained DNA. The velocity again decreases until $R=1.8$ after which it reaches a plateau with a finite velocity (i.e., no neutral complexes). The final mobility shift is somewhat smaller with prestaining, possibly because some catioinic YOYO- 1 is displaced by the CTAB binding, so that the net reduction of the DNA charge is somewhat smaller than with unstained DNA. The experiments illustrated in Fig. 1 indicate that the lack of observable neutral complexes is not due to staining artefacts.

Similar mobility shifts and lack of neutral complexes were observed in linear DNA of sizes 15, 33, 40, and $48 \mathrm{kbp}$ (results not shown), showing that this behavior is a general phenomenon independent of DNA size between 5 and $48 \mathrm{kp}$. The subsequent experiments were performed with $5.4 \mathrm{kbp}$ ФX174-DNA because it has a similar size as the medically relevant plasmids. We used YOYO-1 prestaining because of the higher detection sensitivity with this dye, and the possibility for repetitive scanning of the same gel after different durations of electrophoresis (see below).

\subsection{No complex-dissolution due to dilution}

One possible explanation for the lack of neutral complexes is that once the samples are loaded in the wells, the DNA-surfactant complexes are exposed to the large buffer-reservoir of the gel, so the lipid is free to dissociate since the gel did not contain free surfactant to impose binding equilibrium. To investigate if the DNA-cationic surfactant complexes were undergoing an appreciable dissolution we performed the following experiment. Samples with $R=5$ were prepared and placed in the wells at different times ahead of the electric field application. In this manner the different samples were given time available for potential dissociation by diffusive dilution, covering a time range that included the typical durations for the electrophoresis analysis. Figure 2 shows the resulting gel with samples loaded at different times (between 3 and $0 \mathrm{~h}$ before the field was applied) with lanes 1-4 containing the DNA control samples (no surfactant) and lanes 5-11 containing the DNA-surfactant complex with $R=5$. There is no significant migration difference between the DNA-surfactant samples, in contrast to the progressively faster migration, which would be expected if the DNA gradually loose more and more of the cationic lipid during the waiting. The results in Fig. 2 therefore indicate that there is no dissolution of the complexes due to dilution effects.

The final four samples in Fig. 2 (lanes 12-15) correspond to complexes prepared with the same final concentration of CTAB but from stock solutions whose concentration was below the surfactant CMC ( $0.9 \mathrm{~mm}$ in this case). This last experiment was performed because it has been suggested [29] that when the surfactant is added in the micellar form the DNA condensation will not be as efficient as when added in the monomer form, so the formed complex could retain some negative charges. However, we observed no assignable differences between the two sets of samples (lanes 5, 7, 9, 11, and 12-15).

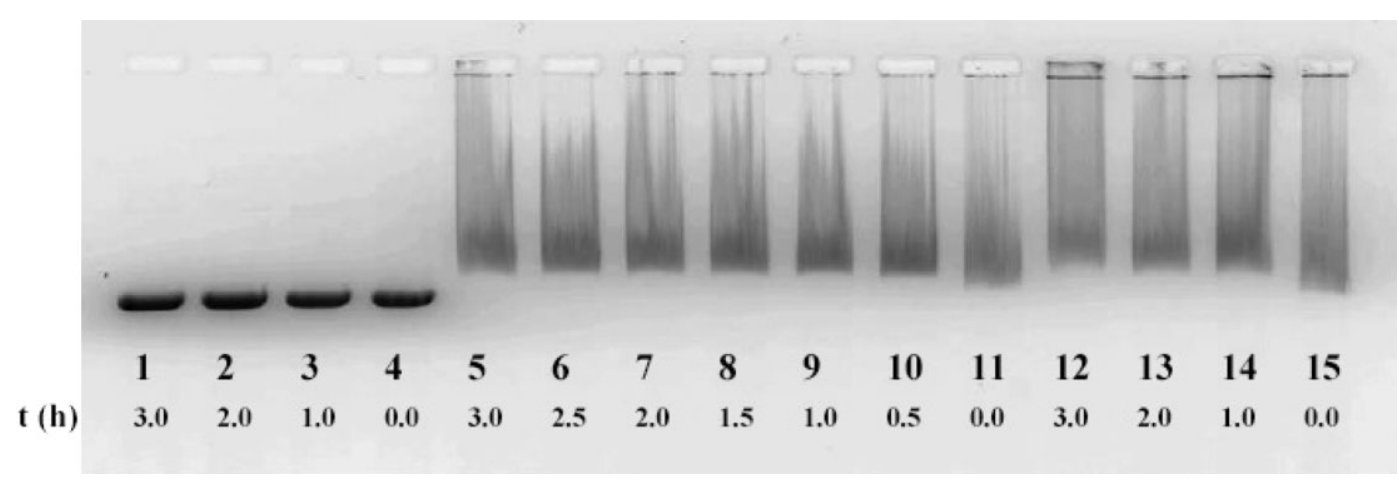

Figure 2. Electrophoretic analysis of potential dissolution of CTAB-DNA complexes due to dilution. Lanes from 1 to 4 contain uncomplexed DNA, lanes 5-11 contain DNA-CTAB complexes at $R=5$ $\left([C T A B]=3.05 \times 10^{-4} \mathrm{M}\right)$. Lanes 12-14 contain DNA-CTAB complexes at the same final CTAB concentration but prepared from CTAB stock solution with concentration below the critical micellar concentration. Time that the samples resided in the wells before the electric field was applied are indicated. Other conditions as in Fig. 1. 


\subsection{Time-resolved electrophoretic velocity in the gel}

A second possible explanation for the lack of neutral DNA-surfactant complexes is that surfactants dissociate during the electrophoretic analysis. To investigate if this occurs, we studied the migration of the complexes as function of time by repetitive scanning of the gel, so that their velocity can be obtained as a function of electrophoresis time. This approach has been used to monitor a migration-induced dissociation of RecA proteins bound to oligonucleotides [30].

We repeated the experiment of Fig. 1 and the gel was scanned not only at the end of the run, but every $0.5 \mathrm{~h}$ for a total of $3.5 \mathrm{~h}$. Figure 3 shows the gel images corresponding to the times $0.5,1.5$, and $2.5 \mathrm{~h}$. The range of mixing ratios $R$ was extended to 200, in order to check that the mixing ratios in Fig. 1 were not too small to reach charge neutralization. As controls for interactions between YOYO-1 and the surfactants we also included samples without DNA but with the same surfactant concentrations as complex samples 6-10 (placed in alternating wells for better comparison). As can be seen in
Fig. 3, both the DNA-containing samples and those DNAfree controls exhibit the same type of bands migrating towards the negative electrode as is observed in Fig. 1. They thus reflect migration of DNA-free surfactant aggregates, consistent with the observation that surfactants added in excess stay in the supernatant [8] and thus are free to migrate on the gel. The binding of YOYO-1 was unexpected since both the dye and the surfactant aggregates are positively charged, but other interactions (such as hydrophobic attractions between the dye molecule and the micelle core) can be expected since our experience is that YOYO-1 adsorbs to a wide variety of surfaces. Association to the surfactant aggregates is expected to give rise to the observed enhanced fluorescence, since this type of dye acquires an increased quantum yield when internal rotation is hindered [31].

Turning the attention to the DNA-surfactant complexes, their migration was further analyzed by evaluating intensity profiles after six different electrophoresis times (Fig. 4), obtained from Fig. 3. Figure 4a shows the first four lanes in Fig. $3(R \leq 1.7)$, and Fig. $4 \mathrm{~b}$ the profiles for the samples with mixing ratios above 1.7 , including the latter a)

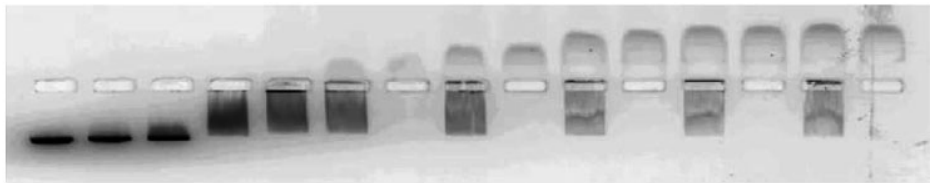

b)

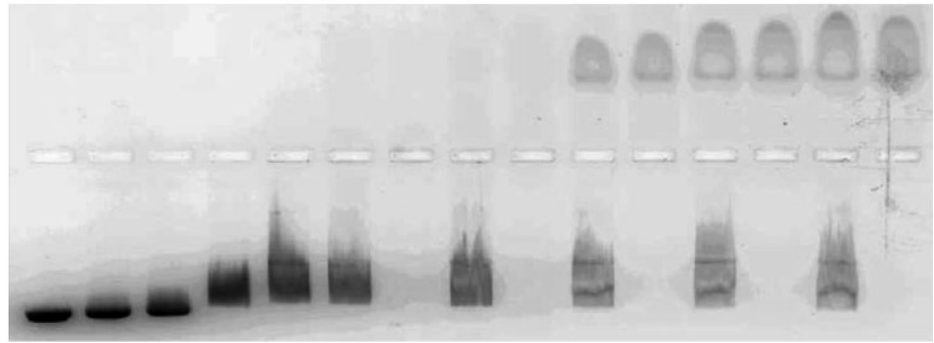

c)

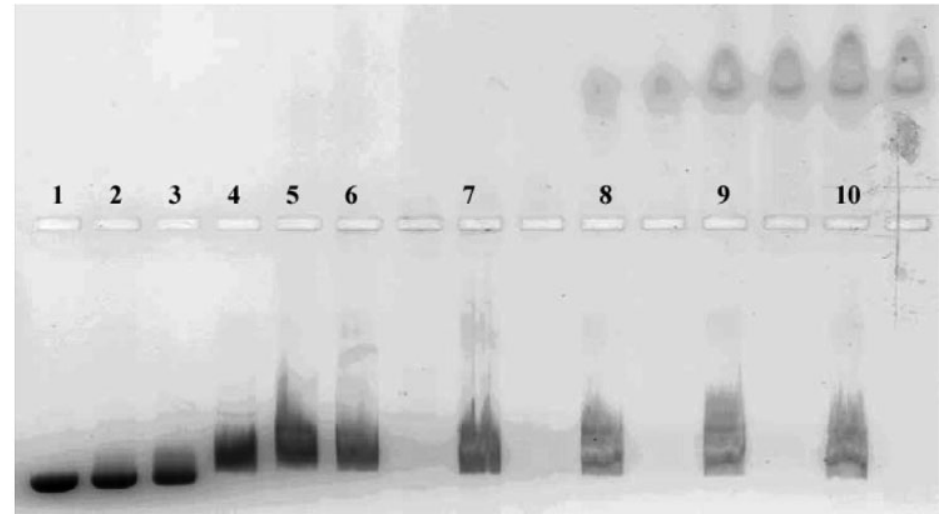

Figure 3. Time-resolved electrophoretic analysis of DNA-CTAB complexes. Complexes between linear $\Phi X 174$ DNA $(20 \mu \mathrm{g} / \mathrm{mL})$ and CTAB at $R$-values (lanes 1-10): 0, 0.5, 0.8, 1.7, 5.0, 13.4, 25.1, 98.8, 150.0, and 200.0. Lanes not numbered correspond to surfactant control samples with the same composition as the one closest to the left but without DNA. Gel was scanned after different durations of electrophoresis (a) $0.5 \mathrm{~h}$, (b) $1.5 \mathrm{~h}$, and (c) $2.5 \mathrm{~h}$. 
again for the sake of comparison. As the migration proceeds, the sample with $R=0.5$ (lane 2) exhibits a delay in the migration (a mobility shift) compared to noncomplexed DNA (R=0, lane 1), and sample $R=0.8$ (lane 3) even more so. At the same time the peak intensity of the samples $R=0.5$ and 0.8 decreases slightly, but this effect is seen also with the noncomplexed DNA and is mainly due to a zone-broadening caused by the migration. The widening (and hence the intensity drop) is somewhat more marked with $R=0.8$ as seen in a weak shoulder appearing on the low-velocity side of the zone. Notably, for $R=1.7$ (which is above charge neutralization) the mobility shift is much more pronounced and the zone is considerably wider than for samples $R=0.5$ and 0.8 (and the noncomplexed DNA). Figure $4 \mathrm{~b}$ shows that for the samples with $R$ equal or superior to 5 we observe the same broad band as in lane $4(R=1.7)$, but also the appearance of a second (but still overlapping) peak on the low-velocity side of the zone.

If the mobility shift in Fig. 4 is analyzed by plotting the final position of the main peak (after $2.5 \mathrm{~h}$ of electrophoresis) versus $R$, one obtains a graph very similar to Fig. 1b, with a plateau velocity again being reached at $R=1.8$ (results not shown). This conventional band-shift analysis can be improved, however, by using the data from all scans. When the position of the main peak is plotted versus the duration of the electrophoresis we obtain a linear dependence for each sample (Fig. 5), and the same is observed for the second slower peak observed for higher $R$-values (Fig. 5, lowest set of curves). This means that the velocity of migration of each band was constant during the electrophoresis, which shows that there is no significant loss of surfactant from the complexes during the migration inside the gel. If the surfactant did dissociate, the velocity is expected to increase with time in a manner reflected by the increase in velocity with decreasing mixing ratio (Fig. 1b). Such an increase in zone velocity was observed when RecA proteins gradually dissociated from complexes with DNA during migration [30].

Importantly, the mobility change seen in a conventional shift plot (Fig. 1b) based on the final zone position does not only reflect the velocity inside the gel (i.e., the slope of the linear fits), but also reflects the fact that the fits do not always pass through the origin. In Fig. 5 a such an effect is observed for samples with $R>1.7$, which indicates that a substantial contribution to the relatively large shifts at those $R$-values is that the complexes are delayed upon entry into the gel compared to noncomplexed DNA. One possibility is that the delay reflects an even lower initial charge at high $R$ (closer to neutrality), which is partly removed by loss of bound lipid as the coils enter the gel. The other possibility that initial complexes contain several DNA molecules and (slowly) break into individual com- a)

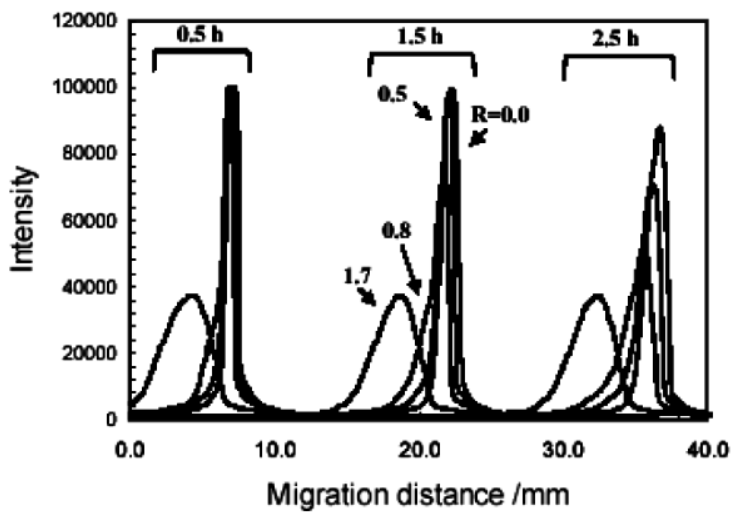

b)

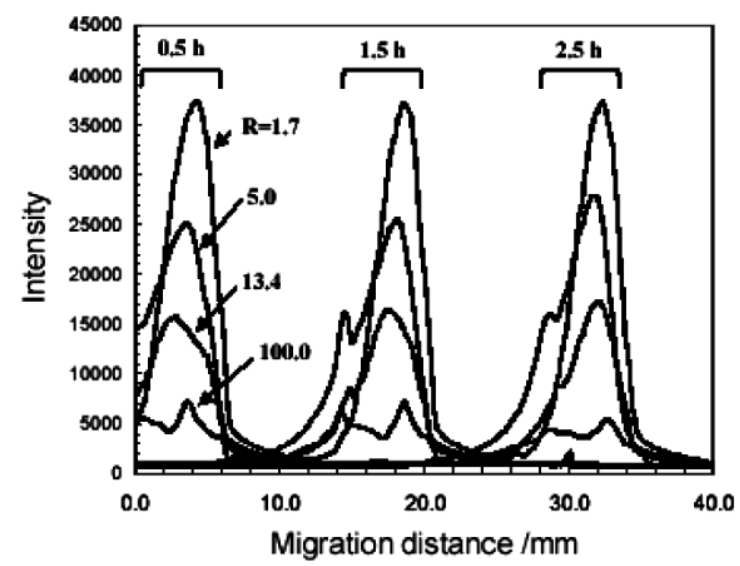

Figure 4. Profile of the bands (fluorescence intensity vs. migrated distance) after different running times (from Fig. 3). (a) Samples corresponding to the first four lanes, open circles: $R=0$, light gray circles: $R=0.5$, dark gray circles: $R=0.8$, black circles: $R=1.67$. (b) Samples from lanes 4-10, open circles: $R=1.67$, gray circles: $R=5.0$, black circles: $R=13.4$, gray triangles: $R=25.1$, open diamonds, crosses, and dotted line (overlapping curves): $R=98.8,150.0$, and 200.0, respectively.

plexes upon entry seems less likely, since results presented below indicate that under our conditions the formed complexes are monomolecular with respect to DNA.

Entry-effects on the apparent mobility can be eliminated by using the slopes, in order to monitor the true complex velocity that the complexes exhibit once inside the gel. Figure $5 b$ shows the in-gel velocity $(v)$ plotted versus the charge ratio $(R)$. The main peak exhibits an initial decrease in velocity followed by an increase to reach a plateau velocity, which is of the same magnitude as the unmodified DNA within experimental uncertainty. The minimum in the velocity as a function of added surfactant (occurring at $R=1.8$ ) shows that the velocity is governed by two opposing factors, in agreement with the prediction 
a)
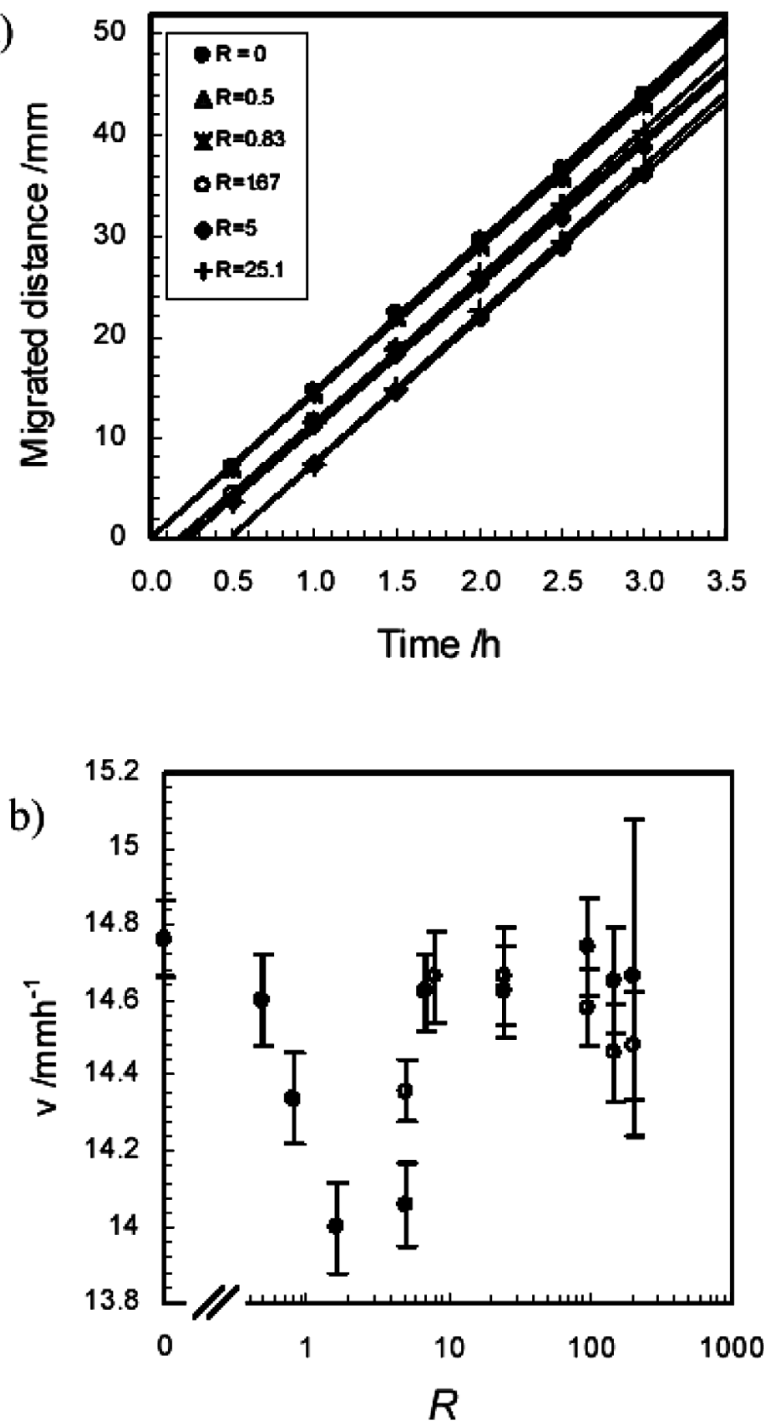

Figure 5. (a) Migrated distance of the bands versus the time of the electrophoresis. Points correspond to the maximum in fluorescence intensity of the bands, including the second slow one, from Fig. 4. A linear fitting was made for each sample. Three top-most lines pass through the origin and correspond to noncomplexed DNA and the first two additions of surfactant, $R=0.5$ and 0.83 , and the lines in the center group correspond to the position of the major peak of the samples at higher surfactant concentrations $(R=1.67,5$, and 25.1) and the lowest set of lines corresponds to the slower peak for the same $R$ values. (b) Plot of the velocity of the complexes in the gel (derived from the slopes of (a)), versus the mixing ratio $R$. Error bars represent uncertainty in the linear fits.

of the Ogston model (Eq. 1). The initial decrease most likely reflects that binding of the cationic lipid initially leads to a decrease in the net charge, i.e., smaller $\mu_{0}$ in Eq. (1). The following increase in velocity probably reflects a decrease in DNA size $R_{\mathrm{s}}$ since additional binding of the cationic surfactant is unlikely to increase the electrophoretic charge $\left(\mu_{0}\right)$ of the complex. A decrease in effective DNA size, occurring at high enough $R$, is consistent with that CTAB is known to induce a coil-to-globule transition, but only at high enough surfactant concentration [7].

\subsection{DNA-CTAB complexes contain one DNA molecule}

In order to model quantitatively the effects on the electrophoretic mobility of CTAB-induced changes in DNA conformation and charge, we first show that the aggregates studied are monomolecular with respect to DNA. This observation allows us to use the well-known sizes and charges of individual native DNA coils, and to invoke the properties of CTAB-induced globules as measured previously by others on individually compacted DNA molecules by fluorescence microscopy. To monitor the number of DNA per complex we performed CTAB condensation on a mixture of T7 and ФX DNA. If the globules are monomolecular, two distinct zones of condensed DNA should be observed, but if on the other hand, complexes contain several DNA molecules, a distribution of complexes also of intermediate sizes is expected. The experiment was performed at $R=5$, where the CTABbinding saturated (Fig. 1b), and at the same total DNA concentrations as used in the experiments here $(0.02 \mu \mathrm{g} /$ $\mu \mathrm{L})$, as well as at twice that value. The results at $0.04 \mu \mathrm{g} /$ $\mu \mathrm{L}$ (Fig. 6) show that the intensity distribution of the mixture is equal to the sum of distributions obtained if the two DNA sizes are run in separate wells, and the same obser-

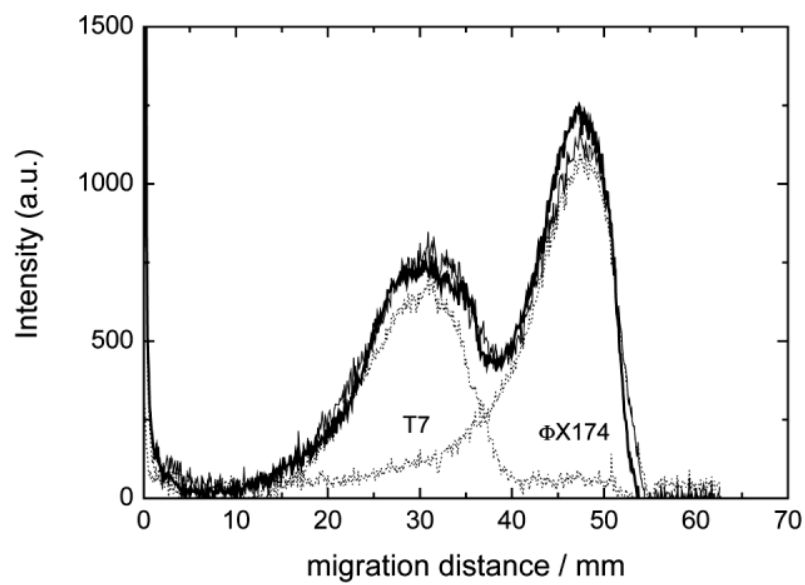

Figure 6. Intensity profiles versus distance along the gel after electrophoretic analysis of complexes between CTAB and $\Phi \times 174$ and T7 DNA, either in a mixture of the two DNA types (thick solid line) or with the two DNA types run in separate lanes (dotted and DNA size indicated). Thin solid curve shows the sum of the dotted curves. In all three samples the concentration of each DNA was $0.02 \mu \mathrm{g} / \mathrm{mL}$ and $R=5$. 
vation was made at half the total DNA concentration (results not shown). We conclude that under our conditions the migrating zones contain individually condensed DNA molecules, as was also noticed by Dauty and Behr [17] when DNA of similar sizes and concentrations were condensed with a cationic lipid.

\subsection{Modeling the electrophoretic mobility of the complexes}

In order to use the Ogston model to estimate the change in mobility when the DNA-size and -charge changes as the globule forms, we first note that in Eq. (1) the average pore radius is $R_{\mathrm{p}}=137 \mathrm{~nm}$ for $1 \%$ agarose [32]. The Ogston-predicted electrophoretic mobility in the gel for the noncomplexed $\Phi \times 174$ DNA is then $0.9 \times 10^{-4} \mathrm{~V} \cdot \mathrm{s} \cdot \mathrm{cm}^{2}$ (Table 1). The reasonable agreement with the experimental value $0.8 \times 10^{-4} \mathrm{~N} \cdot \mathrm{s} \cdot \mathrm{cm}^{2}$ supports that the Ogston model applies under our conditions. For the CTAB-induced (single molecule) globule we assume a fivefold reduction in the effective hydrodynamic radius compared to the coil, as was observed for T4-DNA (166 kbp) [7]. Secondly, the added positive charge at saturated CTAB-binding is estimated to give a resulting net charge of 0.3 e per DNA phosphate, based on a modeling [21] of the binding isotherm [6]. The DNA thus retains some net charge even at saturated CTAB binding because the binding site size is 1.4 DNA phosphate per bound CTAB [7], consistent with a proposed steric crowding of the surfactant head groups on the DNA [6], and with simulations [33] indicating that complete neutralization is not necessary for the globule to form. Our estimation of the globule mobility neglects deviations from the free-draining electrokinetic behavior characteristic of DNA coils. However, a reduction of the free-solu-

Table 1. Electrophoretic mobility for $\Phi X 174$ DNA (5486 bp) in $1 \%$ agarose

\begin{tabular}{lllll}
\hline & $\begin{array}{l}\mu_{0}^{\mathrm{a})} \times 10^{-4} \\
\left(\mathrm{~V} \cdot \mathrm{s} \cdot \mathrm{cm}^{2}\right)\end{array}$ & $\begin{array}{l}R_{\mathrm{s}}^{\mathrm{b})} \\
(\mathrm{nm})\end{array}$ & $\begin{array}{l}\mu_{\text {theory }}{ }^{\mathrm{c}} \times 10^{-4} \\
\left(\mathrm{~V} \cdot \mathrm{s} \cdot \mathrm{cm}^{2}\right)\end{array}$ & $\begin{array}{l}\mu_{\exp }^{\mathrm{d})} \times 10^{-4} \\
\left(\mathrm{~V} \cdot \mathrm{s} \cdot \mathrm{cm}^{2}\right)\end{array}$ \\
\hline Coil & 3.3 & 170 & 0.9 & 0.8 \\
Globule & 1.1 & 35 & 1.0 & 0.8 \\
\hline
\end{tabular}

a) For coil from [37]; for globule estimated from CTAB binding isotherm (see text).

b) For coil $R_{\mathrm{s}}$ is taken as the radius of gyration [20], calculated as $R_{\mathrm{g}}=\left(P L_{\mathrm{c}} / 3\right)^{1 / 2}$ from contour length $L_{\mathrm{c}}=0.34 \mathrm{~nm} / \mathrm{bp}$ and persistence length $P=50 \mathrm{~nm}$. For globule a fivefold reduction is assumed (see text).

c) Calculated according to Eq. (1), using $R_{\mathrm{p}}=135 \mathrm{~nm}$ [32]

d) Calculated from the velocity $14.7 \mathrm{~mm} / \mathrm{h}$ for both noncomplexed DNA and saturated globule at $5 \mathrm{~V} / \mathrm{cm}$ (Fig. 5b) tion mobility to $\mu_{0}($ globule $)=\mu_{0}$ (coil) $\cdot 0.3$ seems reasonable, as it is somewhat weaker but comparable to the reduction by a factor of 0.14 observed for the globule induced by spermidine [34], which is trivalent compared to monovalent CTAB.

Combining the opposite size- and charge-effects, the Ogston model predicts the mobility of the CTAB-induced globule in the gel to differ from that of the coil by less than $10 \%$ (Table 1), which is in agreement with that we observe very nearly the same DNA velocity in the gel at high $R$ as for the noncomplexed coil at $R=0$ (Fig. 5b). These calculations support that the mobility change upon globule formation is the net result of two rather large but opposing effects. Such a near cancellation may explain why with some other cationic lipid condensing agents the mobility for the globule is actually higher than for the noncomplexed DNA [14]. The fact that the velocity passes through a minimum (Fig. $5 \mathrm{~b}$ ) suggests that the charge effect precedes the size effect in the titration, in agreement with that CTAB binding proceeds progressively at $R$-values well below the point of onset of globule formation [6].

\subsection{Release of DNA from the globule}

It has been observed earlier $[8,35]$ that anionic surfactants can be used to release the DNA molecules, which have been compacted by interaction with cationic surfactants. This decompaction behavior was shown to be dependent on the anionic surfactant chain length but showed no dependence on the hydrophobicity of the cationic amphiphile [8]. Here, we use electrophoresis to monitor the dissociation of the complexes, and as an extra twist we use the supercoiled form of the $\Phi$ X174 DNA so that potential nicking could be used to assess the status of the released DNA. Figure 7a shows the image of the gel from a combined association/dissociation study with supercoiled circular DNA. The surfactant association (Fig. 7a, top) resulted in the same kind of mobility shift (and zone broadening) as was observed for the linear form (Fig. 1), again pointing to the generality of our observations. The DNA sample contains trace amounts of relaxed circular and linear DNA, which migrate more slowly (square 4 in lane 2, Fig. 7a) than the supercoiled form under the present conditions (as determined by the photocleavage behavior [36]).

The DNA-cationic surfactant sample corresponding to $R=5.0$ (where compaction was deemed complete) was then subjected to a titration series with increasing concentration of the anionic surfactant SDS (Fig. 7b). For mixing ratios, $S_{R}=\left[S^{-}\right] /\left[S^{+}\right]$of anionic and cationic surfactants below 0.9 , the anionic surfactant leads to several 
a)

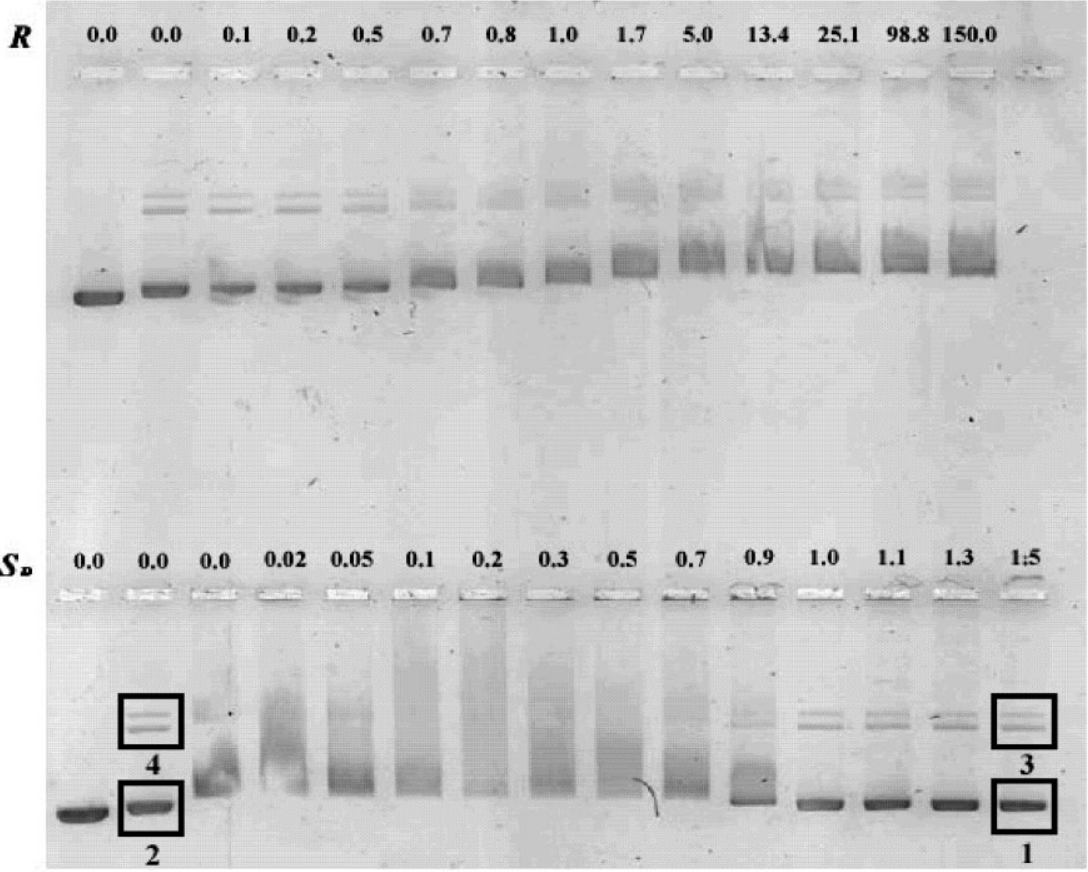

c)

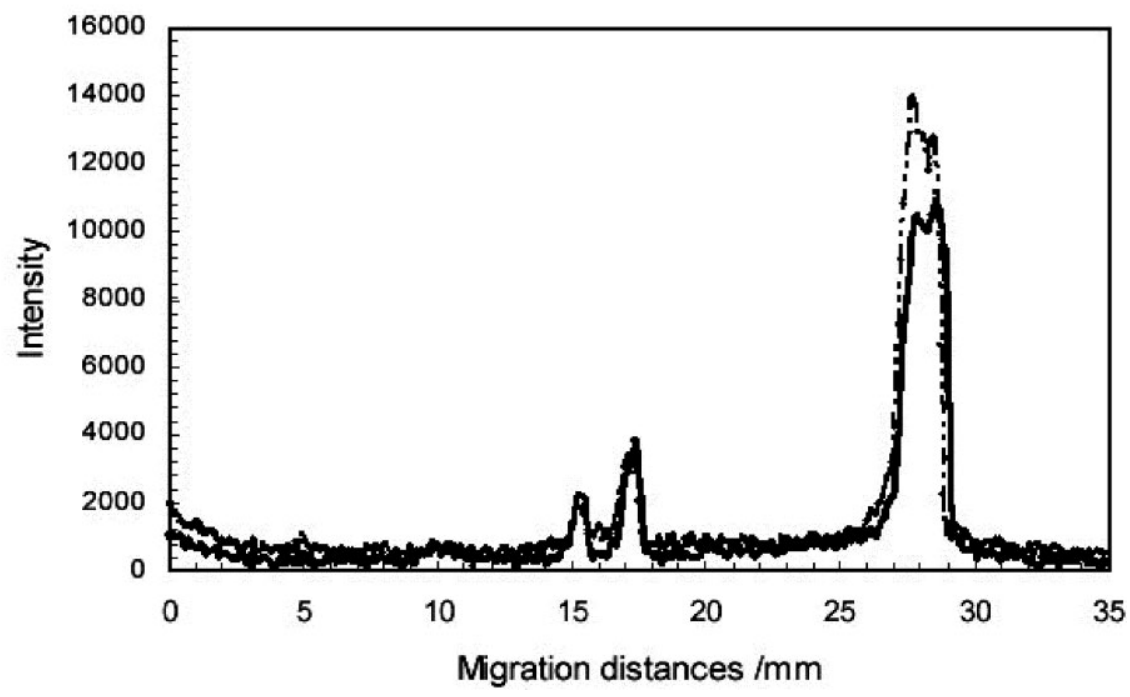

Figure 7. (a) Electrophoresis of the complexes between circular ФX174 DNA and CTAB and the dissociation of the complexes corresponding to sample (b) $R=5$ with the addition of SDS. $R$ represents, as before, the mixing ratio between cationic surfactant and DNA, while $S_{R}$ corresponds to the charge ratio of negative and positively charged surfactants. Values are presented in the figure for each sample. Prestaining with YOYO. (c) Profile of bands plotted as the intensity of the fluorescence versus the migration distances. Thicker line corresponds to pure ФX174 DNA, the second lane in (b). Thinner line corresponds to the last lane, representing the dissociation of DNA-CTAB complexes $(R=5)$ with the addition of SDS, for $S_{R}=1.5$. wide zones, suggesting a broad distribution of complexes with respect to size and/or charge during the dissolution process. Here the focus will be at mixing ratios above $S_{R}=0.9$ where a zone of the same velocity and shape as noncomplexed DNA (lane 2) starts to appear, and above $S_{R}=1.0$ the DNA molecules seem to be fully released since both the position and width of the zone are close to those observed with noncomplexed DNA. Similar reversible behavior was obtained with linear $\Phi$ X174 and $\lambda$ DNA (results not shown), hence successful release does not depend on the molecular weight or topological state of the DNA molecules.
The required surfactant mixing ratio we observed for complete release is in good agreement with the one previously obtained using fluorescence microscopy [22], in spite of the concentrations of both DNA and cationic surfactant being about 100-fold lower in the microscopy experiments. The decompaction of DNA was shown to occur through the formation of mixed surfactant aggregates, which was described [22] as a critical micellar concentration for the surfactants' mixture $\left(\mathrm{CMC}_{\text {mixt }}\right)$. Whereas the single-molecule nature of fluorescence microscopy allowed us to determine the onset of this dissociation when the first DNA molecules are decom- 
pacted, the present ensemble-averaging technique rather monitors the mixing ratio $S_{R}$ that is required for complete dissociation of the CTAB-DNA complexes.

From Fig. $7 \mathrm{~b}$ we can estimate how large fraction of the DNA is recovered intact after the process of compaction and release, since the supercoiled form can be separated from the relaxed circular and linear molecules that would potentially result from single-strand nicking and doublestrand breaks, respectively. The ratio between the supercoil and other two forms was about $82 / 18$ for the noncomplexed DNA (squares 2 and 4, respectively, in Fig. 7a), while it was $78 / 22$ (squares 1 and 3 , respectively) after this sample had undergone aggregation and dissociation by oppositely-charged surfactants. This means that about $95 \%$ of the supercoiled DNA was recovered in this native form after the release. Considering the sensitivity of the assay (one nick per $5486 \mathrm{bp}$ is detected) this observation adds considerabe support to the conclusion [15] that the process of compaction of DNA by cationic surfactants is reversible upon the addition of anionic surfactants, as based on circular dichroism, EB binding, and DNAse protection. The absence of nicking is particularly important if compaction release is to be used for plasmid purification.

Dissociation of the DNA-CTAB complexes was also performed using the anionic surfactant SOS (results not shown). This surfactant, due to its shorter hydrophobic chain, is much less efficient than SDS; only at a mixing ratio $S_{R}$ of about 10.0 did the first dissociated DNA molecules become visible and the majority of them were recovered to the solution only for $S_{R}$ equal to 30.0. These results are also similar to those previously obtained by fluorescence microscopy [22].

This work was supported by grants from Praxis XXI (PRAXIS/BD/21227/99), the Fundação para a Ciência e Tecnologia (FCT) (project Sapiens PCTI/99/QUI/35415), and the Swedish Research Council for Engineering Sciences (VR 98-638). Heidi Talvitie is thankfully acknowledged for experimental assistance and good discussions.

Received July 26, 2004

\section{References}

[1] Wagner, E., Cotten, M., Foisner, R., Birnstiel, M. L., Proc. Natl. Acad. Sci. USA 1991, 88, 4255-4259.

[2] Ogris, M., Steinlein, P., Kursa, M., Mechtler, R., Wagner, E., Gene Ther. 1998, 5, 1425-1433.

[3] Kimura, T., Yamaoka, T., Iwase, R., Murakami, A., Macrom. Biosci. 2002, 2, 437-446.

[4] Yoshikawa, K., Adv. Drug Del. Rev. 2002, 52, 235-244.

[5] Köping-Höggård, M., Mel'nikova, Y. S., Vårum, K. M., Lindman, B., Artursson, P., J. Gene Med. 2003, 5, 130-141.
[6] Mel'nikov, S., Segeyev, V., Yoshikawa, K., J. Am. Chem. Soc. 1995, 117, 9951-9956.

[7] Mel'nikov, S., Sergeyev, V., Yoshikawa, K., J. Am. Chem. Soc. 1995, 117, 2401-2408.

[8] Dias, R., Mel'nikov, S., Lindman, B., Miguel, M. G., Langmuir 2000, 16, 9577-9583.

[9] Guillot, S., McLoughlin, D., Jain, N., Delsanti, M., Langevin, D., J. Phys.: Condens. Matter 2003, 15, S219-S224.

[10] Trewavas, A., Anal. Biochem. 1967, 21, 324-329.

[11] Dutta, S., Jones, A., Stacey, M., Biochim. Biophys. Acta 1953, 10, 613-622.

[12] Wagner, K., Harries, D., May, S., Kahl, V., Rädler, J. O., BenShaul, A., Langmuir 2000, 16, 303-306.

[13] Eastman, S. J., Siegel, C., Tousignant, J., Smith, A. E., Cheng, S. H., Scheule, R. K., Biochim. Biophys. Acta 1997, 1325, 41-62.

[14] Lobo, B. A., Davis, A., Koe, G., Smith, J. G., Middauagh, C. R., Arch. Biochem. Biophys. 2001, 386, 95-105.

[15] Bhattacharya, S., Mandal, S. S., Biochemistry 1998, 37, 7764-7777.

[16] Crook, K., McLachlan, G., Porteous, D. J., Gene Ther. 1996, 3, 834-839.

[17] Dauty, E., Behr, J. P., Polym. Int. 2003, 52, 459-464.

[18] Viovy, J. L., Rev. Mod. Phys. 2000, 72, 813-872.

[19] Slater, G. W., Rousseau, J., Noolandi, J., Turmel, C., Lalande, M., Biopolymers 1988, 27, 509-524.

[20] Holmes, D. L., Stellwagen, N. C., Electrophoresis 1990, 11, 5-15.

[21] Spink, C. H., Chaires, J. B., J. Am. Chem. Soc. 1997, 119, 10920-10928.

[22] Dias, R. S., Lindman, B., Miguel, M. G., J. Phys. Chem. B 2002, 106, 12608-12612.

[23] Gao, X., Huang, L., Biochemistry 1996, 35, 1027-1036.

[24] Xu, Y., Szoka, F. C., Biochemistry 1996, 35, 5616-5623.

[25] Zelphati, O., Szoka, F. C., Proc. Natl. Acad. Sci. USA 1996, 93, 11493-11498.

[26] Carlsson, C., Jonsson, M., Åkerman, B., Nucleic Acids Res. 1995, 23, 2413-2420.

[27] Carlsson, C., Larsson, A., Jonsson, M., Electrophoresis 1996, 17, 642-651.

[28] Lleres, D., Clamme, J. P., Dauty, E., Blessing, T., Krishnamoorty, G., Duportial, G., Mely, Y., Langmuir 2002, 18, 10340-10347.

[29] McLoughlin, D. M., O’Brien, J., McManus, J. J., Gorelov, A. V., Dawson, K. A., Bioseparation 2001, 9, 307-313.

[30] Svingen, R., Takahashi, M., Åkerman, B., J. Phys. Chem. B 2001, 105, 12879-12893.

[31] Carlsson, C., Larsson, A., Jonsson, M., Albinsson, B., Norden, B., J. Phys. Chem. 1994, 98, 10313-10321.

[32] Stellwagen, N. C., Electrophoresis 1992 13, 601-603.

[33] Dias, R. S., Pais, C. C. A. A., Miguel, M. G., Lindman, B., J. Chem. Phys. 2003, 119, 8150-8157.

[34] Yamasaki, Y., Teramoto, Y., Yoshikawa, K., Biophys. J. 2001, 80, 2823-2832.

[35] Mel'nikov, S. M., Dias, R., Mel'nikova, Y., Marques, E. F., Miguel, M. G., Lindman, B., FEBS Lett. 1999, 453, 113-118.

[36] Åkerman, B., Tuite, E., Nucleic Acids Res. 1996, 24, 10801090.

[37] Stellwagen, N. C., Gelfi, C., Righetti, P. G., Biopolymer 1977, $42,687-703$. 